\title{
LA «BIBLIA FILOSÓFICA» Y LA REFORMA: EL CASO DE JOHN LOCKE
}

\author{
POR \\ MONTSERRAT HERRERO ${ }^{1}$ \\ Universidad de Navarra
}

\section{RESUMEN}

La transición de la Escritura como una obra sobrenatural a un mero texto es algo que tiene que ver con el movimiento reformado, sin embargo, no se puede atribuir como tal a la Reforma, sino sobre todo a la corriente filosófica ilustrada, nutrida de las convicciones que movilizó la Reforma. El artículo analiza esta corriente en uno de sus predecesores: John Locke. Su obra contribuyó a generar la «Biblia académica», como contrapuesta a la «Biblia escriturística». En la obra de Locke se pone de manifiesto una dialéctica entre el carácter sobrenatural de la Escritura y la consideración de la razón como revelación natural, que la conduce a numerosas aporías. De ahí que la bibliografía haya considerado a John Locke alternativamente calvinista, latitudinario, arminiano, sociniano o unitariano. El artículo presenta estas aporías y procura una evaluación de su aportación a la cuestión de la interpretación bíblica, que es indisociable de su fe, de su epistemología y de su teoría del lenguaje.

PALABRAS CLAVE: Locke; Reforma; Biblia; Interpretación bíblica.

\section{THE «PHILOSOPHICAL BIBLE» AND THE REFORMATION: THE CASE OF JOHN LOCKE}

\begin{abstract}
The transition of Sacred Scripture from a supernatural text to a mere secular text can be related to the Reformation, but we cannot say that it was the work of the protestant Reformation. Moreover it was the result of the enlightened biblical criticism practiced by some philosophers, as is the case of John Locke. Locke's work contributes to the construction of the "Academic Bible» as opposed to the "Biblical Scripture». In his writings we can detect a kind of dialectic between the supernatural character of the Sacred Scriptures and the consideration of reason as natural revelation that leads to some paradoxes. Because of that the literature has considered Locke's position, as been alternatively Calvinist, Arminian, Socinian, Latitudinarian or Unitarian. This article deals with these paradoxes and evaluates the original Lockean thesis on Biblical interpretation, which is inseparable of his faith, his epistemology and his theory of language.
\end{abstract}

KEY WORDS: Locke; Reformation; Bible; Biblical Interpretation.

Cómo CITAR ESTE ARTículo / CITATION: Herrero, M. 2018. «La 'Biblia filosófica' y la Reforma: el caso de John Locke». Hispania Sacra 70, 141: 75-83. https://doi.org/10.3989/hs.2018.007

\author{
Recibido/Received 09-05-2017 \\ Aceptado/Accepted 09-06-2017
}

\section{LA BIBLIA REFORMADA}

Frente a lo que la opinión común piensa, la Reforma no defiende una interpretación subjetiva de la Biblia. ${ }^{2}$ Ciertamente los textos de los reformadores tienen como consecuencia, al menos histórica, una ruptura entre la interpretación de la Escritura y la tradición de la Iglesia católica, pero no se puede decir que de ellos se deduzca

1 mherrero@unav.es / ORCID iD: http://orcid.org/0000-00018949-3742

2 Lutero 1883-1929: 98. necesariamente una completa libertad de conciencia. ${ }^{3}$ Para Lutero y también para Calvino la Escritura «impone» su verdadera interpretación al sujeto debidamente formado. No se puede decir, por tanto, que el sujeto "construya» el significado de la Escritura. Como señala García-Alonso,

3 Cf. Balaguer 2017: 171-192. El artículo recoge resumidamente la posición luterana y calvinista sobre la interpretación de la Escritura. Pone de manifiesto la desconfianza de las mediaciones humanas (175); y, sin embargo, la cierta cercanía respecto de la relación entre Escritura y tradición que puede reconocerse tanto en el planteamiento reformado como en el católico (185). 
«es muy importante no confundir el rechazo protestante del magisterio de la Iglesia católica con la defensa de la interpretación subjetiva de la Biblia». ${ }^{4}$ Para Lutero y Calvino no hay nada oscuro en la Escritura, de modo que las enseñanzas de la Biblia han de ser tenidas por primeros principios evidentes a partir de los cuales se generan los razonamientos. Son las conclusiones resultantes de la argumentación lo que es interpretable, pero no los mismos principios revelados. La Escritura es, por tanto, su propio intérprete. Ella tiene su gramática propia, la gramática de la fe. Ciertamente, y esto puede llegar a ser un problema, las palabras que emplea son las mismas que las que se emplean en el lenguaje ordinario. En cualquier caso, la teología es para Lutero, la disciplina de la "página sagrada», de un modo similar a como lo fue en el periodo medieval. ${ }^{5}$ Tanto Lutero como Calvino predicaban la necesidad de una adecuada preparación para la interpretación de la Biblia y no lo que se entendió en la modernidad por «libre examen». Como señala García-Alonso, para ambos el examen remite a la independencia de juicio sobre la interpretación que haga la Iglesia de la Escritura, pero no al comentario individual.

Estas ideas tuvieron su correlato político. Efectivamente Lutero pretende sustituir la teoría de los dos poderes por la de los dos reinos con formas de ejercicio del gobierno totalmente diferentes. ${ }^{6}$ Calvino también desarticulará la potestad eclesiástica, sin embargo, apostará por una fórmula en la que Dios como fuente de toda autoridad exige del magistrado civil una función sagrada. Los magistrados son instrumentos de Dios. ${ }^{7}$

La idea de que la Escritura es su propio intérprete es una idea reformada que pasa a la crítica bíblica ilustrada en la forma que adquiere en Thomas Hobbes, Baruch Spinoza y John Locke. ${ }^{8}$ Para referirse a esta doble tradición Legaspi establece una distinción entre lo que él denomina la «Biblia académica» y la "Biblia escriturística». ${ }^{9}$ Dentro de

\section{García Alonso 2008: 56-57.}

5 Sigo aquí la interpretación de la tesis del libro de Hagen 1993, quien se propone restituir una lectura auténtica de los comentarios a la Escritura de Lutero basados en los manuscritos y no en el Weimar Ausgabe que contiene los «prejuicios» de la exegética del siglo XIX. E mismo Lutero habla contra el método interpretativo cuando dice que la teología de Pablo ha de ser aplicada al tiempo presente, pero no interpretada. Hagen, por tanto ve los trabajos de Lutero como una enarratio bíblica medieval, más que como un comentario interpretativo moderno, que es como han venido siendo considerados.

6 Cf. Abellán 2008: IX-XXXVII.

7 Cf. García-Alonso 2016: IX-LXIX.

Prieto López 2017: XIII: «Locke hace suyo el biblismo integral que se expresa en la máxima protestante, de origen luterano, de la sola Scriptura, que el calvinismo también había aceptado».

Cf. Legaspi 2010. El libro está dedicado a cómo llega a existir la «Biblia académica» como una empresa post-confesional en el siglo XVIII. Aunque muchos interpretan el nacimiento de esta tradición como una labor cooperativa con la tradición de la Biblia escriturística que respeta la autoridad confesional, lo cierto es que en la llustración, que es el momento en el que nace esta tradición, se emplea el criticismo moderno como una profilaxis contra el fundamentalismo. Con ella aparece la dialéctica entre historia y revelación, lo secular y lo sagrado, ciencia y religión. El libro de Legaspi que introduce la distinción mencionada se centra en la obra de Johann David Michaelis (1717-1791), quien en su opinión es el gran responsable de la inauguración de esta tradición junto otros contemporáneos como Christian Gottlob Heyne, Johann Gottfried Herder, Immanuel Kant. Michaelis era un luterano. Legaspi no cita a Locke directamente, aunque parece que sí implícitamente cuando comenta: «The Works of Spinoza, Hobbes, Simon, Peyrère, and others la primera, yo distingo la "Biblia filosófica», que es la que se corresponde con el privilegio de la racionalidad sobre otras áreas disciplinares en el estudio de la Biblia como texto. Muchas son las diferencias entre la pretensión de los reformadores en este punto y los filósofos ilustrados. ${ }^{10}$ La razón filosófica y no la fe se convirtió en la última ratio interpretativa de la Escritura. ${ }^{11}$ Este fue el credo de John Locke; y esto a pesar de que su creencia en el carácter sobrenatural de la Escritura no sufriera nunca menoscabo. ${ }^{12}$ No se puede decir sin más que Locke fuera ante todo un protestante y sólo secundariamente un filósofo. Es una tesis diversa la que se va a intentar justificar aquí: no es ante todo un protestante, aunque fuera una persona profundamente religiosa, ni es ante todo un filósofo, aunque ciertamente es uno de los padres del empirismo, sino sobre todo un teólogo político que profesó en la Iglesia de Inglaterra. ${ }^{13}$

\section{RAZÓN Y FE: CERTEZA Y ESCEPTICISMO ENTRE LA LEY DE LA NATU- RALEZA Y EL ENSAYO SOBRE EL ENTENDIMIENTO HUMANO}

En opinión de Ashcraft el Ensayo sobre el entendimiento humano ha de ser leído como una confirmación de la fe de Locke, aunque no consiga una reconciliación definitiva entre su cristianismo y su filosofía. Esta fue su intención al escribirlo y este debe ser el punto de partida de una adecuada interpretación, aunque el contexto de lectura de los últimos siglos, con la perspectiva de un estrato temporal más amplio, lo haya interpretado como una radical salida del statu quo filosófico del momento. ${ }^{14}$ Tal y como aparece en la Epístola al lector, su propósito es estudiar hasta qué punto los principios de la moral y de la religión revelada son capaces de dirigir las acciones humanas. Finalmente, como señala Marshall, en ese Ensayo Locke fue incapaz de dar una respuesta satisfactoria a la vida después de la muerte o al castigo por el pecado, dado su agnosticismo respecto de la sustancia y de los atributos de Dios. ${ }^{15} \mathrm{~A}$ esto hay que añadir la dificultad de reconciliar la confianza en la razón

bear witness to the fact that the kind of philological, critical and analyses of the Bible associated with biblical modern studies were already well attested by the latter part of the 17th Century». Legaspi 2010: 4. Lo tiene muy en cuenta sin embargo Michael J. Lee como precedente europeo del escepticismo bíblico americano, que es en lo que centra su libro Lee 2013: 11. Brad Gregory incluye también a Locke en al menos tres de sus seis genealogías del mundo moderno que pesan en nuestro presente, el cual se caracteriza en su opinión tanto por un rechazo, como por una variada apropiación de diferentes elementos del cristianismo medieval a través de la Reforma. Locke fue uno de los pensadores influyentes en esa línea, tanto en lo que supuso un control de las iglesias, como en lo que Gregory denomina una secularización del conocimiento. Cf. Gregory 2012.

Cf. Balaguer 2017: 175. La posición reformada es que la razón debe desaparecer ante la fe; en Calvino esto admite matices, desde luego.

11 Cf. Herrero 2017: 31-49.

12 En las últimas décadas ha habido una considerable producción dedicada a la rehabilitación del Locke teólogo: Dunn 1969; Waldron 2002; Spellman 1988; Marshall 1994; ibídem 2006; Nuovo 2011; Mitchell 1993; Schwartzman 2005: 678-705; Pritchard 2014; Herrero 2015. La tradición contraria sería la que incluye obras como: Pangle 1988; Euben 1999; Kahn 2011; Sommerville 1992; Zuckert 2002.

13 La impronta política en la filosofía de John Locke está justificada en Herrero 2015. También en ibídem 2016: 83-109.

14 Cf. Ashcraft 1969: 194-223.

15 Cf. Marshall 1994: 453. 
humana, que se entrevé en su ensayo sobre La ley de la naturaleza escrito muchos años antes, con el hedonismo y el escepticismo de su Ensayo sobre el entendimiento humano, como señala von Leyden. ${ }^{16}$ La mayor tragedia a la que se enfrenta todo hombre es que quedan fuera del conocimiento cierto las realidades más relevantes para la vida para las cuales se requiere una guía segura. En opinión de Marshall, es justamente esa difícil reconciliación lo que lleva a Locke, consciente de su incapacidad para elaborar una teoría del conocimiento moral que suponga una orientación para la vida política en el Ensayo sobre el entendimiento humano, a acudir a la interpretación de la Escritura y a escribir finalmente La razonabilidad del cristianismo. ${ }^{17}$ Pero es justamente ahí donde se vuelve a abrir paso la confianza en la razón. Veamos este intricado movimiento.

Ya en su escrito sobre Ley de la naturaleza habla Locke de cuatro tipos de conocimiento - por inscripción, por tradición, por el sentido y por revelación divina- para poner de relieve que en lo tocante al descubrimiento de la ley de la naturaleza sólo cuenta el sentido. ${ }^{18}$ Solamente podemos dar asentimiento a lo que obtengamos por experiencia, sensación y reflexión. A través de ese medio se puede tener certeza de muy pocas cosas, las que nos da el conocimiento intuitivo, el conocimiento demostrativo y el conocimiento sensible de ideas simples. Más allá de esto, lo que tenemos son distintos grados de probabilidad fundados bien en la conformidad con nuestra propia experiencia, a lo cual denomina razón ${ }^{19}$; o bien en el testimonio de la experiencia de los otros, a lo que denomina fe o creencia. ${ }^{20}$ Dentro de la segunda habría que considerar la revelación, como su capítulo más relevante, porque en su caso el testigo es Dios mismo. Un aspecto particular que afecta a la fe es su transmisión, es decir, la tradición. Lógicamente conocemos por fe, caso de que exista una revelación, aquello que no es accesible a la luz de la razón. De algún modo Locke admite que las jurisdicciones de la fe y la razón son diferentes, aunque se apresura a decir que no hay contradicción ni puede haberla entre fe y razón: "La fe no es otra cosa sino un firme asentimiento por parte de la mente, el cual, si está bien regulado, según es nuestro deber hacerlo, no puede otorgarse a nada que no esté apoyado en buena razón, de

16 Sin embargo, no dudará Locke hasta el final de sus días en la capacidad de conocer la ley de la naturaleza, como se puede leer en Locke 1956: II. 28. $\S 5$ y $\S 13$. Así lo constata quien editó por primera vez el ensayo La ley de la naturaleza. Cf. Von Leyden 1956: 26: «In my view, Locke tended in his later years to regard the notion of a law of nature as a mere premise of his thought, as something he believed in but barely investigated. The reason for this attitude, I think, is to be found in difficulties he had in reconciling the notion of this law with some of his mature doctrines. For instance, the development of his hedonistic views and his philosophy of language in the Essay had made it difficult for him to attempt a full exposition of natural law or even to believe in it whole-hearted».

17 Marshall 1994: 388.

18 Locke 2007: 18.

19 Locke 1956: IV. 17. § 2: «la facultad que indaga los medios y que los aplica debidamente para descubrir la certidumbre en el uno y la probabilidad en el otro, es la que llamamos razón».

20 Locke 1956: IV. 17. § 7. "como hay muchas cosas acerca de las cuales tenemos nociones muy imperfectas, o ninguna, y como hay otras cosas de cuya existencia pasada, presente y futura nada podemos saber por vía del uso natural de nuestras facultades naturales y se sitúan por encima de la razón, estas cosas, digo, cuando han sido reveladas, son el asunto propio de la de la fe». suerte que no puede serle opuesto». ${ }^{21}$ En cualquier caso, tiene que haber una buena razón para el asentimiento ${ }^{22}$, es decir, que la razonabilidad de la fe se reconduce a la autenticidad o no de la revelación y a la fiabilidad de la tradición por la que llega esa revelación, aunque parece que Locke sólo considera la posibilidad de revelaciones directas y no a través de testigos. En cualquier caso argumenta en los dos sentidos.

En cualquier caso, el ejercicio de la racionalidad siempre se hace necesario como apoyatura de la fe. Todo hombre que ha recibido una revelación ha de comunicarla sirviéndose de las nociones comunes que otros no entenderán más que si también las han experimentado por vía de sensación o de reflexión. Pero además, en segundo lugar, es que nunca podemos estar seguros de si algo es una verdadera revelación, más que si no contradice la razón. Aquí Locke aboga por una teología natural, por una capacidad de pensar las realidades sean naturales o sobrenaturales, que pueda discernir en el conflicto de discursos interpretativos. ${ }^{23}$

En último término, esta es su convicción, el conocimiento que tenemos por revelación nunca puede ser tan seguro como el procedente de nuestra razón: "La garantía de ser una revelación es menor que la garantía que ofrecen los sentidos». ${ }^{24}$ Por eso, una revelación que esté en contra de lo que nos dice la razón tiene muchas posibilidades de no ser verdadera. Por otra parte, como él mismo señala, la razón es ya una originaria revelación natural. ${ }^{25}$ En el caso de que hubiera contradicción entre una supuesta revelación de Dios y nuestro entendimiento, entonces, deberíamos dudar de que sea una verdadera revelación de Dios, porque Dios mismo no puede ir en contra de los principios del conocimiento que él mismo nos ha proporcionado. ${ }^{26}$

21 Locke 1956: IV. 17. § 24.

22 Ibídem: IV. 18. § 8. «Sin embargo, siempre es a la razón a la que toca juzgar acerca de si en verdad se trata de una revelación, y sobre el significado de las palabras en que ha sido concebida. Es cierto que si alguna cosa que sea contraria a los principios evidentes de la razón, y al conocimiento manifiesto que la mente tiene de sus propias ideas claras y distintas, pasa por ser una revelación, en tal caso es preciso escuchar la voz de la razón, como en asunto que es de su competencia».

${ }^{23}$ Cf. Nuovo 2002: XV-LVII. Una afirmación elogiosa de la teología como ciencia se encuentra en Locke 1992: § 23.

24 Locke 1956: IV. 18. § 4.

25 Locke 1956: IV. 19. § 4: «La razón es la revelación natural, por donde el eterno Padre de la luz y manantial de todo conocimiento les comunica a los hombres esa porción de verdad que ha puesto al alcance de sus facultades naturales. La revelación es la razón natural, aumentada por un nuevo acervo de descubrimientos comunicados inmediatamente por Dios, y de los cuales la razón garantiza su verdad, por el testimonio y las pruebas que da acerca de que proceden de Dios. De suerte que quien proscribe la razón para admitir la revelación extingue la luz de ambas, y hace, poco más o menos lo mismo que si persuadiera a un hombre a sacarse los ojos, para mejor recibir, por medio de un telescopio, la remota luz de la estrella invisible».

26 Locke 1956: IV. 18. $\S 6$ : "Hasta este punto un hombre tiene derecho a recurrir a su razón, y debe prestarle oído, hasta en el caso de una revelación inmediata y original, que se supone que se le hace a él mismo. Pero, por lo que toca a todos aquellos que no pretenden ser beneficiarios de una revelación inmediata, y a quienes se exige que acaten y reciban las verdades que han sido reveladas a otros hombres, las cuales por tradición escrita $u$ oral les han sido comunicadas, tienen mucha más necesidad de la razón, y es ella la única que puede inducirnos a recibirlas. Porque, como los asuntos de fe solamente son de revelación divina, y nada más eso, la fe (tomando esa palabra por lo que comúnmente llamamos fe divina) no tiene nada que ver con ninguna 
La revelación a la que accedemos por tradición presenta más problemas. Claramente, el testimonio de un porcentaje amplio de testigos aumenta la probabilidad de que una revelación sea verdadera. En opinión de Locke, también la cercanía temporal de los testigos. Cuanto más remotos sean los testimonios, menor será su valor probatorio. ${ }^{27} \mathrm{~A}$ esto le llama una inversión de la regla de la probabilidad. La tradición no es fuente de conocimiento suficiente para la fe, de modo que sólo resta esperar algo de la revelación y de la razón. Sólo en el caso en que la razón no tenga con claridad algo como verdadero y la revelación añada algo a esa ignorancia, ha de aceptarse la revelación sin dudar de ella. ${ }^{28}$ Si la religión no diera cabida a la razón en el tratamiento de sus asuntos entonces se generarían toda clase de fantasías. ${ }^{29}$

\section{EL PROBLEMA DEL LENGUAJE, LA INTERPRETACIÓN Y LA INFALIBILIDAD}

La cuestión de la Revelación de Dios se enfrenta con un problema añadido al de la autenticidad del testigo y es que Dios habla a los hombres de un modo diferente a como habla la realidad sensorial. A Dios no podemos verle y sentirle. Lo único que tenemos de él son palabras. Y las palabras no están conectadas naturalmente con las ideas y con la realidad, sino que se generan por la costumbre de conectar ciertas palabras con ciertos significados. ${ }^{30}$ Dios usa en su comunicación las mismas "palabras imperfectas», cuyo sentido último sólo la razón puede determinar. ${ }^{31}$

$\mathrm{Si}$ es cierto que las palabras son un medio tan poco seguro, entonces la interpretación racional es el único modo de leer un texto, incluidas las Escrituras. La voluntad de Dios está revestida de palabras y en cierto sentido oculta en ellas. ${ }^{32}$ Por eso, dirá Locke, hay tantos

proposición, salvo con aquellas que se suponen de revelación divina. De suerte que yo no puedo comprender cómo, quienes sostienen que la revelación es el único objeto de la fe, puedan decir que es asunto de fe, y no de razón, creer que tal o cual proposición, que se encuentra en este o aquel libro, es de inspiración divina, a no ser que sepan por revelación que esa proposición, o todas las que se encuentran en ese libro, han sido comunicadas por una inspiración divina».

27 Locke 1956: IV. 16. § 10: «tratándose de las verdades tradicionales, cada alejamiento debilita la fuerza de la prueba; y mientras mayor sea el número de manos por donde ha pasado la tradición, menor será la fuerza y la evidencia que recibe de ellas. Me pareció necesario advertir esto, porque he notado que algunos hombres practican justamente lo contrario, y son aquellos que consideran que una opinión adquiere mayor fuerza a medida que envejece».

28 Locke 1956: IV. 18. § 9.

29 Ibídem: IV. 18. § 11: «habiendo sido imbuidos los hombres en la opinión de que no deben consultar a la razón en materias religiosas, por más contradictorias que sean al sentido común y a todos los principios del conocimiento, han dado rienda suelta a su fantasía y a sus naturales inclinaciones supersticiosas, $y$, por ello, han sido inducidos a aceptar unas opiniones tan extrañas y a practicar unas ceremonias tan extravagantes en asuntos religiosos, que un hombre sensato no puede menos de asombrarse en sus locuras».

30 Locke 1956: III, 2, § 1.

31 Ibídem: IV, 18, § 8 y IV, 19, § 4.

32 Ibídem: III, 9, § 23: «Aun cuando todo lo escrito en el texto sea infaliblemente verdadero, sin embargo, el lector puede, o mejor dicho, no puede menos de ser muy falible en entenderlo. Ni es de sorprender que la voluntad de Dios, cuando revestida en palabras, esté expuesta a esa duda y a esa incertidumbre que inevitablemente acompaña a esa clase de comunicación (...)». y tan variados comentaristas de las Sagradas Escrituras y por eso es necesario ser caritativo con las diferentes opiniones relativas a esos textos tan antiguos y en los que es tan difícil dirimir cuál pueda ser su verdadero sentido. ${ }^{33}$ No hay una sola interpretación válida de las Escrituras. La infalibilidad papal se presenta como un acto de arrogancia intolerable.

La lucha contra la infalibilidad papal es uno de los primeros caballos de batalla de Locke. Efectivamente el texto Infallibility de 1661 es uno de sus primeros escritos, aunque permaneció inédito durante toda su vida. En aquel momento en el que Europa se batía en guerras sectarias originadas, al menos aparentemente, por discusiones sobre el credo, la Iglesia católica con su idea de infalibilidad parecía el único refugio serio. Sin embargo, Locke ofrece en ese primer ensayo dos argumentos contra esta solución: el primero se fundamenta en la idea de providencia divina y reza: si la infalibilidad papal fuera necesaria para la salvación, Dios la hubiera previsto, pero no fue así, sino más bien es un desarrollo posterior de la época apostólica. El segundo argumento se basa en la idea de que Dios no actúa de un modo superfluo y dado que los hechos históricos contravienen la necesidad de la supuesta infalibilidad de la Iglesia, puesto que al comienzo no fue así, hemos de suponer que es algo superfluo, no específicamente provisto por Dios. ${ }^{34}$

En el Ensayo sobre el entendimiento humano Locke añade dos argumentos más contra la infalibilidad. El primero es que de la coyuntural conveniencia histórica de la existencia de un árbitro en la tierra para conseguir la paz, no se deduce una necesidad de principio, ni un dogma. Del mismo modo entonces se podría deducir la necesidad de la infalibilidad individual, mostrar su conveniencia y entonces concluir que Dios hizo las cosas de ese modo. ${ }^{35}$ El segundo es que la tradición no puede ser usada para justificar la corrección de la interpretación, como hacen quienes defienden la infalibilidad de la Iglesia coronada en el papa, precisamente porque la tradición no es una fuente de verdad suficiente. Si la Iglesia en la persona del papa no es infalible, entonces, ¿quién interpreta? La respuesta de Locke en Infallibility es la siguiente:

Está fuera de duda que la interpretación de la Sagrada Biblia deriva en parte del estudio, en parte de la razón y, finalmente, en parte del Espíritu Santo que ilumina las mentes de los hombres, pero el más seguro intérprete de la Escritura es la Escritura misma y sólo ella es infalible. ${ }^{36}$

33 Cf. Locke 1956: III, 9, § 22. Cf. Wensheimer 1993: 45, habla del «monological individualism» de Locke. En su opinión la idea de tolerancia en Locke se deriva de un individualismo lógico, es decir, de una presencia de elementos anti-hermenéuticos en su epistemología. Efectivamente el conocimiento cierto es poco y las palabras de la Escritura ocultan su significado, pero además Locke presupone que el entendimiento humano no se puede hacer cargo e interpretar lo que otros seres humanos piensan, dicen y escriben. $C f$. Wensheimer 1993: 238. Locke no es suficientemente hermeneuta porque no tiene esperanza de poder resolver los conflictos de un modo definitivo dialógicamente, de ahí que prevea el dispositivo de la tolerancia. No cae en la cuenta que el diálogo es una forma de saber y no sólo de comunicación. Cf. Wensheimer 1993: 45.

34 Locke 2002a: 69-73.

35 Locke 1956: I, 4, § 12

36 Locke 2002a: 72. 
EL PROYECTO DE HACER RAZONABLE EL CRISTIANISMO: LA CONSTRUCCIÓN DE LA VERDADERA RELIGIÓN

No cabe duda de que Locke está proponiendo un concepto de religión verdadera: aquella que entroniza a la razón como juez del ámbito de lo sobrenatural. De la verdadera religión sólo se puede estar cierto a través de una interpretación del texto sagrado. En la última etapa de su vida Locke se lanza a esa tarea en la escritura de la Racionalidad del cristianismo. ${ }^{37}$ Es en la razón implícita en la Escritura en donde se puede encontrar la conciliación de las diferentes confesiones y es también a través de ella como se pueden asegurar los principios de la religión y la moralidad.

Como señala Nuovo, la estrategia de Locke bien puede haber sido influida por el libro de Socino De Auctoritate Scripturae Sacrae (1570), a pesar de que Locke rechaza esta posibilidad en Vindication of the Reasonableness of Christianity y en su Second Vindication of the Reasonableness of Christianity, defendiéndose de la acusación de socinianismo de parte de John Edwards. ${ }^{38}$ En su libro, Socino defiende la autoridad de la Escritura por encima de cualquier otra: cualquiera que crea que la religión cristiana es superior por razón de la evidencia de su superioridad, no puede dudar de la autoridad de la Escritura. De ahí que lo único importante sea ratificar la autenticidad de la Escritura y de su interpretación. Uno de los caminos para llegar a ello propuestos por Socino será la comparación de los diferentes evangelios para mostrar cómo en su armonización se hace posible una narrativa coherente; otro camino consiste en certificar que los evangelistas fueron realmente testigos válidos de la vida de Cristo; un tercero propone estudiar cómo la palabra profética y las promesas mesiánicas del Antiguo Testamento llegan a cumplimiento en los evangelios. En opinión de Nuovo, las dos obras teológicas mayores de Locke, The Reasonableness of Christianity y $A$ Paraphrase and Notes on the Epistles of St Paul usan estas estrategias interpretativas. ${ }^{39}$

Por otro lado, Nuovo constata la influencia del Tratado teológico-político de Spinoza en la obra de Locke. Locke anota en su Biblia, conservada en la Bodleian Library, textos del tratado de Spinoza entre 1672 y 1675. En particular, parece que Locke asume la idea de Spinoza de que los judíos eran ignorantes acerca de la causas naturales de los acontecimientos atribuyendo toda causalidad de cualquier evento a la acción divina. También adopta una opinión spinoziana en lo relativo a la inspiración profética. Aunque rechaza su naturalismo y se opone a la negativa de considerar el carácter sobrenatural de la Biblia. ${ }^{40}$ También Locke debía estar bien informado sobre las doctrinas deístas sobre la autoridad de las Escrituras, puesto que en A Vindication of the Reasonableness of Christianity Locke defiende su libro arguyendo que su objetivo al escribirlo

37 Como señala Leopoldo José Prieto López en su estudio preliminar a la traducción española siguiendo el prólogo de Locke en la Second Vindication of the Reasonableness of Christianity, el motivo inmediato de la escritura de La razonabilidad fue clarificar la naturaleza de la justificación y la fe, recurriendo para ello únicamente a la luz de la Escritura. Prieto López 2017: XI-CXXXII.

38 Para una discusión completa de estas obras cf. Nuovo 2012: $X I X-L X X V I I$.

39 Cf. Nuovo 2011: 12.

40 Cf. Ibídem: 55. era persuadir a los deístas de las ventajas de la revelación cristiana y de la autenticidad de su interpretación. ${ }^{41}$ En ese sentido, a diferencia de Spinoza, Locke ofrece una prueba de la autoridad divina de la Escritura que no disminuye su fundación racional.

Según Israel, ni Socino, ni Spinoza, ni el deísmo fueron las únicas influencias en la escritura de La razonabilidad del cristianismo. En su opinión fue profundamente influido por el debate entre Limborch y Orobio de Castro sobre la validez de la doctrina mesiánica. ${ }^{42}$ Para Limborch, lo único que exige el cristianismo como doctrina necesaria es creer que Jesús es el Mesías; aparte de esto, la doctrina cristiana revela su origen divino por medio de la santidad de sus preceptos.

En efecto, en The Reasonableness of Christianity as Delivered in the Scriptures $(1695)^{43}$ Locke distingue entre el núcleo de la fe, lo que cualquier cristiano está obligado a creer en todo tiempo, y la formulación de la fe, que es variable históricamente. Es a esta formulación a la que hay que referir los distintos sistemas teológicos o "sistemas de divinidad", como él los denomina, que compiten entre sí por dar una explicación acorde con nociones de carácter filosófico. Locke piensa que ningún sistema teológico puede agotar la verdad de la fe. El individuo siempre es libre en la elección de sus creencias. La misión de los "sistemas de divinidad» está en aportar pruebas para la racionalidad de un determinado credo y ayudar al individuo a elegir entre ellos. Desde esta concepción se comprende la importancia de la cuestión de la tolerancia en el pensamiento de Locke. En el prefacio de The Reasonableness of Christianity, Locke avisa de que la insatisfacción que le han producido todos los sistemas de divinidad que ha encontrado hasta el momento es lo que le mueve a hacer su propia interpretación de la Escrituras. Lo que Locke busca es articular una nueva forma de cristianismo que esté por encima de todo sectarismo, una forma comprensiva en la que quepan todas las interpretaciones disponibles hasta el momento. Nuovo denomina a este intento una "teología liberal». ${ }^{44}$ No lo

41 Locke 2002b: 213.

42 En oposición a Orobio, un crítico judío de la religión cristiana, Limborch, Leclerc y Locke afirmaban que la verdad cristiana debe ser probada por la razón basada en la evidencia. Para un debate sobre esta cuestión cf. Israel 2001: 464-470.

43 Locke 2017. J. Marshall consigna que hubo varias ediciones de esta obra. Esta y A Paraphrase and notes on the Epistles of St. Paul de 1707 fueron las dos investigaciones que realizó Locke al final de su vida. Las dos fueron consideradas por sus contemporáneos como heréticas. Como señala Marshall 1994: 453-454, esto debió producir en Locke una gran ansiedad, teniendo en cuenta que algunos de sus contemporáneos eran ejecutados por ser antitrinitarios, como fue el caso de Thomas Aikenhead y teniendo en cuenta que en la Inglaterra de aquel momento se publicó una Blasfemy Act contra el antitrinitarismo en 1697. Marshall considera que en su última obra Locke era, en privado, unitariano. Entre 1660 y 1690 cambió de ser trinitariano y de creer fuertemente en el pecado original a tener opiniones completamente heterodoxas en ambos temas. En cualquier caso Locke fue un miembro de la Iglesia de Inglaterra desde su restablecimiento en 1662 hasta el final de su vida.

44 Nuovo 2011: 53-54: «lt should be evident to anyone who has read John Locke's mature theological writings that his primary motive as an author was to articulate a form of Christianity acceptable to all Christians not predisposed by sectarian interests, one that would prove persuasive to all impartial readers, whether confidently Christian or not, a form of religion that to his mind was not his own invention, but which represented faithfully the idea of Christianity perspicuously presented in Scripture, especially in the New Testament. The title of 
hace, desde luego, sin una aproximación metódica. A lo que había concluido en Infallibility, añade años más tarde en el Ensayo sobre la racionalidad del cristianismo:

[La Escritura debe ser comprendida] en el sentido directo y claro de las palabras y expresiones, que es el sentido que puede suponerse que tenían en la boca de quienes las pronunciaban, los cuales las usaban con arreglo al lenguaje de la época y país en el que vivían, sin darles sentidos tan cultos, artificiales y forzados como los que se inventan y se imponen a las palabras en la mayoría de los sistemas teológicos, de acuerdo con los conceptos que a cada cual le han inculcado. ${ }^{45}$

De este modo de interpretar se deduce que las verdades reveladas que todo cristiano debe creer son muy pocas en opinión de Locke «ese es el núcleo de la fe»:

[...] no toda verdad de las Escrituras inspiradas forma parte de aquellas verdades que, por la ley de la fe, se exige que sean explícitamente creídas para la justificación [...] cualquier otra parte de la Escritura que Dios no haya constituido parte necesaria de la ley de la fe (sin un asentimiento real a la cual él no reconocerá a nadie como creyente) puede ser ignorada por un hombre sin poner en riesgo su salvación por imperfección de la fe. ${ }^{46}$

El fundamento de la fe es simplemente creer en el Mesías: «Esta es la gran afirmación que fue entonces objeto de controversia acerca de Jesús de Nazaret: si era el Mesías o no; y la respuesta afirmativa era lo que distinguía a los creyentes de quienes no creían $»{ }^{47}$ Para convencer a los hombres de esto realizó todos los milagros. ${ }^{48}$ La segunda gran creencia fundamental es la resurrección. ${ }^{49}$

Con esta premisa, contrapone la ley de las obras a la ley de la fe. Ciertamente si no hubiera habido ley de las obras, tampoco podía haber ley de la fe, pues no hubiera hecho falta. La ley de las obras es esa ley que requiere obediencia perfecta sin ninguna relajación ni reducción. En esa ley la justificación viene por el cumplimiento de cada tilde..$^{50}$ La ley de las obras fue entregada por Moisés. La ley de la naturaleza está incluida en ella. La ley de la fe, sin embargo, fue entregada por Jesucristo. Por la ley de la $\mathrm{fe}^{51}$ se permite que la fe supla el defecto de la obediencia plena, de modo que «los creyentes

The Reasonableness of Christianity as deliver'd in the Scriptures is an announcement of that intention. Accordingly, he employed a theological method that is biblical and expository rather than speculative and dogmatic, and in this endeavor he was confident not only of the divine authority of Scripture but also of Scripture's capability to disclose its paramount meaning». Nuovo afirma que Locke se encuentra en la tradición del «liberalismo teológico» que comienza con Erasmo y que incluye a Castellio, Hugo Grocio, Richard Hooker y el conjunto del "Tew Circle». E mismo Lelio Socino se puede incluir en esta lista. Nuovo 2011: 47. Según señala Marshall 1994: 391-392, Locke conocía bien la doctrina de Socino. En el momento de su muerte poseía una gran colección de los principales autores socinianos y del mismo Socino, que había comprado en torno a 1680. Entre los estudiosos, realmente se le ha incluido en muy diferentes grupos: calvinistas, latitudinario, arminiano, sociniano o unitariano.

45 Locke 2017: 5.

46 Ibídem: 231-232.

47 Ibídem: 25.

48 Ibídem: 60.

49 Ibídem: 152

50 Ibídem: 18.

51 «De modo que, la ley de la fe es en suma, que cada cual crea lo que Dios le exige que crea como condición de la alianza que hace con él, y que no dude en el cumplimiento de sus promesas». Ibídem: 23. son admitidos a la vida y a la inmortalidad como si fueran justos». ${ }^{52}$

Si bien la parte moral de la ley de Moisés obliga a los cristianos y a todos los hombres en todos los lugares siendo así que es coincidente con la ley de la naturaleza-, la parte civil y ritual de la ley entregada por Moisés no obliga a los cristianos, aunque para los judíos sea parte de la ley de obras. La ley de la fe no consta de una parte ritual. ${ }^{53}$ Ahora bien, insiste en que esta fe no sustituye a las obras, puesto que en la Escritura está por todos lados el mandato del arrepentimiento:

El arrepentimiento es un sincero dolor de nuestros delitos pasados y una sincera resolución y empeño de conformar todas nuestras acciones a la ley de Dios, en cuanto está en nuestro poder. [...] Estas dos cosas, fe y arrepentimiento, es decir, creer que Jesús es el Mesías y una vida dedicada al bien, son las condiciones indispensables de la nueva alianza, las cuales han de ser cumplidas por todos aquellos que quieran alcanzar la vida eterna. ${ }^{54}$

La vida buena es obediencia a la ley; ahora bien, contará la fe como obediencia donde la fragilidad y debilidad hizo fallar en las obras. Lo que salga de esto es o superstición o un interés político de las diferentes facciones teológicas.

Uno de los más importantes resultados interpretativos de su método en esta obra es la doctrina sobre el pecado de Adán. ${ }^{55}$ Por el pecado de Adán entró la muerte en el mundo, no, sin embargo una debilitación de la naturaleza humana que el género humano hereda de él, como muchos creen. Ninguno es castigado más que por sus propios hechos, por tanto, Jesucristo simplemente trajo la restitución, es decir, recuperó la inmortalidad para el hombre. La gracia no es necesaria para salvarse y consiguientemente tampoco los sacramentos, ni, por tanto, la casta sacerdotal y la Iglesia en su conjunto.

Este presupuesto teológico en la particular interpretación que hace Locke, subyacía ya en los Dos tratados sobre el gobierno civil al defender sus tesis anti-patriarcalistas, por un lado, y la idea de la igualdad natural de todos los hombres por otro. Ahora en esta obra de madurez explicita con total claridad el presupuesto teológico y las consecuencias que de él se derivan sobre todo en lo referente al culto en relación con la religión verdadera. ${ }^{56}$ En la visión de Locke todo lo referente al ceremonial y los templos, pertenece al politeísmo idolátrico y politeísta del mundo antiguo. Y el cristianismo ha venido muy principalmente a depurar eso. Locke pasa por alto los pasajes de la Escritura en que Jesús instituye el nuevo rito. $Y$ se centra fundamentalmente en el pasaje de la samaritana en el que Jesús señala que la verdadera adoración es en espíritu y verdad. Lo único que Dios requiere de ahora en adelante es la aplicación de la mente y sinceridad de corazón. Si no hay ritos, tampoco es necesaria la Iglesia. Si hay ritos entonces, como él dice, «hay casta sagrada» y hay «templo». De su interpretación se

\footnotetext{
52 Ibídem: 20.

53 Sobre la cuestión del culto cf. Herrero 2015: 34-46.

54 Locke 2017: 148.

55 Ibídem: 4-11.

56 La discusión sobre este punto está extensamente desarrollada
} en Waldron 2002. 
deduce que la Iglesia es una mera asociación entre otras..$^{57}$ Todo lo que tiene en contra de las iglesias, lo tiene, sin embargo, a favor de la religión sobrenatural. La revelación de Dios ha sido necesaria para el progreso de la humanidad:

A la vista de lo poco hecho hasta ahora a este respecto, parecería que es una tarea demasiado ardua para la razón establecer por sí sola todos los aspectos de la moralidad sobre sus verdaderos fundamentos, con una luz clara y persuasiva. $Y$ desde luego, es un camino más seguro y más corto para la comprensión del vulgo, que es la mayor parte de la humanidad, que alguien, manifiestamente enviado por Dios y que viene de él con visible autoridad, les imponga como rey y legislador sus deberes y les exija obediencia, antes que dejar que esto se les aclare después de largas y a veces intrincadas deducciones de la razón. ${ }^{58}$

La Escritura es para Locke el timón de la orientación de su conducta, como lo muestra el siguiente texto de su respuesta al obispo de Worcester en relación con sus críticas al Ensayo sobre el entendimiento humano:

La Santa Escritura es para mí, y siempre lo será, la guía constante de mí consentimiento; y siempre la escucharé como conteniendo la verdad infalible relativa a las cosas del más alto interés [...] y yo condeno ahora mismo y tranquilamente cualquier opinión mía, que se me muestre como contraria a la Sagrada Escritura. ${ }^{59}$

El círculo se cierra donde empezó. Esta es la primera gran aporía: Locke pretendió salvar las disputas teológicas a través de la recta razón en su capacidad de descubrir la ley natural y el ordenamiento político, pero finalmente nada de eso era tan seguro como él pretendió al comienzo, de modo que al final de su vida recala de nuevo en la interpretación de la Escritura como segura guía para las limitaciones del hedonista y el escéptico. Pero la Escritura seguía siendo el pantanoso terreno de las disputas. A pesar de ello, Locke se da cuenta de que la religión se hace tanto más necesaria como guía, cuanto menos capaz es la razón de conocer la realidad y de orientar desde ese conocimiento las pasiones humanas. El difícil equilibrio entre la racionalidad y la fe religiosa se da cita en la interpretación. Solo desde una interpretación "adecuada» se pueden salvar los conflictos políticos. Y esta es la segunda aporía: La liberación política de las disputas teológicas comienza por la aceptación de una interpretación bíblica que desactive la lucha en el terreno civil entre la religión y la política. Ciertamente, la resolución de esta segunda, depende a su vez de la primera.

\footnotetext{
57 Locke 2002c: 80.

Este breve manuscrito es una anotación del año 1682. Consiste en una nota escrita al hilo del libro I, capítulo 15 del libro Laws of Ecclesiastical Polity de Richard Hooker. En él Locke reflexiona acerca de la distinción que ahí aparece entre la iglesia como una asociación sobrenatural y la iglesia como una mera asociación natural o social. La doctrina de Locke no coincide con la de Hooker. El interés de Locke es limitar el poder eclesiástico, de ahí que aunque se base en Hooker, concluye de su reflexión algo muy diferente, a saber, que la iglesia es una asociación voluntaria. Esto significa que: a. la pertenencia no puede ser obligada; b. la pertenencia se funda en el consentimiento; c. el único motivo para constituir una iglesia es el principio del culto público de Dios. Es la misma idea que aparecerá años más tarde en la Primera carta sobre la tolerancia y en La conducta del entendimiento.

58 Locke 2017: 201.

59 Citado por Ashcraft 1969: 223.
}

\section{LIBERACIÓN A TRAVÉS DE LA INTERPRETACIÓN DE LAS ESCRITURAS}

Según Locke, el uso de la razón hace libres a los hombres, mientras que el creer en principios y autoridades como medio de justificar las propias acciones les hace esclavos. ${ }^{60}$ Locke parece querer decirnos que cualquier "sistema de verdad" es un "sistema de poder», de ahí que liberación de la razón del yugo del dogmatismo de la autoridad y de la tradición se traduce en una liberación política. Este tipo de liberación está impedida por dos obstáculos principales: el primero es la infalibilidad en lo relativo a la interpretación, que concede poder a la Iglesia; y, el segundo es a la tradición, que apoya el gobierno patriarcal tanto en la Iglesia como en la sociedad civil. La interpretación por la razón disminuye el peso de ambos obstáculos confiriendo poder a una razón autónoma y, de ese modo, a una libertad mayor. Desde el punto de vista institucional, por tanto, los enemigos de la libertad fueron la Iglesia católica y la monarquía de los Estuardo.

Ciertamente Locke posicionó a la Iglesia católica, asociada entonces a la monarquía, frente al republicanismo liberal fundado en la libertad de pensamiento; sin embargo, esta enemistad no se hizo extensible a la religión en general. La secularización del espacio público como tal está ausente en la argumentación de Locke. ${ }^{61}$ Para Locke la religión es importante para fundar un orden político. ${ }^{62}$ Justamente la exclusión de la monarquía y el catolicismo, en su opinión, es necesaria para abrir el camino a la religión verdadera, que viene de la mano de la "Biblia filosófica», que él mismo contribuye a construir con su interpretación de la Escritura, como hemos visto. ${ }^{63}$

Con la distinción entre la ley de la fe y la ley de las obras, Locke permite la distinción de los dominios político y religioso. La fe cristiana, que es universal, debe ser independiente de las circunstancias políticas, que son siempre históricas. La fe cristiana es un hecho relevante, no sólo para la historia de

60 Locke 1956: I, 4, § 25.

61 Como señala Nuovo en Nuovo 2011: 98: "Locke's place in the Enlightenment must be represented in the light of his Christian commitments». Sobre su cristianismo dice: "I do not think it is saying too much to assert that he belongs as much to the Christian tradition as does Augustine of Hippo, Thomas Aquinas, or John Calvin. He is a principal founder of modern liberal Christianity. Locke's philosophy, then, should be viewed as a point of orientation for a historical reconstruction of this tradition whose first flowering occurred in the seventeenth century in Holland and England [...]. In sum, Locke's Christian philosophy gave substance and direction to English liberal theology». Nuovo 2002: XVIII-XIX. Para Nuovo las diferencias entre la tradición teológica liberal de Locke y el agustinismo y el calvinismo son: «the consequences of Adam's sin for his posterity, the punishment of the damned, and the competence and duty of mankind to fulfil the moral conditions of salvation». Nuovo 2002: XVIII-XIX.

62 Dunn 1969: 99: «Jesus Christ (and Saint Paul) may not appear in person in the text of the Two Treatises but their presence can hardly be missed when he comes upon the normative creaturely equality of all men in virtue of their shared species membership». Una discusión sobre la necesidad del punto de vista teológico para concebir la igualdad en el planteamiento de Locke en los dos Tratados sobre el gobierno civil se encuentra en Waldron 2002.

63 Israel estaría de acuerdo con esta afirmación, pues para él la diferencia más importante entre los sistemas de Spinoza y Locke es que la tolerancia tal como la concibe John Locke tiene que ver con la libertad de culto y la discusión teológica, mientras que la tolerancia de Spinoza es esencialmente filosófica, republicana y explícitamente anti-teológica. Israel 2001: 265. 
la salvación, sino también para la historia de la humanidad. El cristianismo lleva consigo un progreso en la racionalidad en comparación con las religiones paganas. En opinión de Locke este elemento revolucionario del cristianismo hace posible un republicanismo universal. Su razonabilidad del cristianismo es una defensa de una religión universal que confiere el poder a la razón y que constituye una teología política que propugna el republicanismo liberal como un medio universal de gobierno.

El ideal de esta defensa, sin embargo, puede ser contrastado por su posición en los primeros escritos sobre la tolerancia, donde en caso de expandirse una religión no verdadera, el magistrado debe ejercer su poder, como si fuera un representante de Dios. En efecto los Two Tracts on Government $(1660)^{64}$, son una defensa de la autoridad casi absoluta en su confrontación con las religiones que pudieran ser inadecuadas. Los consecutivos escritos de Locke sobre la tolerancia muestran una posición más moderada, pero aun con todo, mantiene a lo largo de toda su obra la idea de que el juicio sobre la religión, si es o no verdadera, si es o no nociva para el bien público, depende de la prerrogativa del magistrado. ${ }^{65}$ Sin duda, La razonabilidad del cristianismo, construye un terreno de cierta objetividad para ese juicio. En cualquier caso Locke subordina la religión al juicio político y en este sentido hace de la esfera política la última instancia de apelación en la tierra. Este argumento es reforzado hasta sus últimos días como se puede ver en $A$ Paraphrase and notes on the Epistles of St. Paul. ${ }^{66}$

Tan claros son los enemigos políticos y tan clara la vocación política de la vida de Locke, ejercida fundamentalmente a través de Lord Ashley, por mucho que renunciase en su vida a todos los cargos políticos, que no es difícil sospechar de la pretendida neutralidad tanto en el desarrollo de su filosofía política como en su interpretación de la Escritura. ${ }^{67}$

64 Locke 1967: 120. Para una discusión de esta cuestión ver Herrero 2016: 83-109.

65 Sobre la posición «moderada» de Locke es interesante el juicio de Hahn y Wiker 2013: 465-486. Su tesis es que él no sacó las conclusiones de la interpretación bíblica que había sembrado, pero puso los cimientos para que otros los sacaran y la historia hizo su tarea. De hecho, en su opinión, su interpretación de San Pablo como un autor sembrado de errores que pertenece a un contexto histórico del que no se puede desligar, es mucho más radicalmente historicista que la posición de Spinoza. Así señalan: "Locke is best understood as a moderate radical. As a moderate radical, he was the bearer of the very radicalism at the heart of his thought. At the same time he pulled away from it, he drew attention to it and allowed it to enter the mainstream of respectable thought». Hahn y Wiker 2013: 486.

66 Cf. Locke 1824.

67 Así lo reconocen Hahn y Wiker 2013: 2, donde se habla de los «political commitments inherent in the core foundations of the method itself». O también: «by politizacion we mean the intentional exegetical reinterpretation of Scripture so as to make it serve a merely political, this-wordly (hence secualr) goal». Hahn y Wiker 2013: 9. Es fundamenta en opinión de Hahn y Wiker ir a los orígenes del criticismo bíblico y no comenzar su historia en el siglo XVIII. En particular sobre John Locke: Hahn y Wiker 2013: 425-463. En el mismo sentido Reventlow 1985: 285: "[t]he whole of Locke's scriptural exegesis is a further example of the way in which at this period exegesis is not done for its own sake but because of the normative validity of scripture for quite specific questions of political life or in the associated search for the foundations of social ethics which would serve as a criterion in current controversies over the form of state and society in England». En el mismo sentido Herrero 2015.

\section{CONCLUSIÓN}

Locke sigue el principio reformado de la sola scriptura: la Escritura lleva en sí su propia interpretación. Sin embargo, no acepta otro de los grandes principios de la Reforma, a saber, que la Escritura tiene "su propia gramática» dependiente de la fe. Más bien la razón es el principio hermenéutico prioritario y casi absoluto. De ahí la posibilidad de hablar de una "Biblia filosófica». Como se ha podido percibir en el artículo, un etiquetado confesional de su Biblia filosófica es complejo. Comparte estrategias interpretativas socinianas, acaba siendo unitariano en lo relativo a la doctrina trinitaria, podría considerársele latitudinario por su teología liberal que se afana por estar por encima de todo sectarismo dando importancia a la racionalidad y a la práctica del cristianismo; coincide con el arminianismo en el enfrentamiento al rigorismo calvinista. Quizás haciendo una síntesis podría calificarse como un calvinista heterodoxo que fue hasta sus últimos días un miembro de la Iglesia de Inglaterra.

Lo cierto es que, aunque su Biblia no es la Biblia protestante, las consecuencias que Abellán prevé para la modernidad a partir de los desarrollos de Lutero, se cumplen eminentemente en John Locke: en primer lugar, des-clericalización de la sociedad; en segundo lugar, la negación de una esfera sagrada independiente de la actividad mundana; en tercer lugar, la ruptura de la unidad religiosa y con ello la neutralización de la religión misma. ${ }^{68}$ Yo añadiría a estas consecuencias la entronización del juicio político sobre las religiones.

Efectivamente, esa ratio filosófica no parece desinteresada, como se muestra en los escritos sobre la tolerancia -que no eran objeto de este artículo- ${ }^{69}$ Tampoco se esconde su interés en su posición anticatólica en la Exclusion Crisis. Sin duda su interpretación de la Escritura es acorde con los fines políticos que Locke persigue, tanto teóricos la instauración de un republicanismo liberal secular- como estratégicos en su contexto histórico - la restauración de la monarquía orangista protestante- En este sentido es un teólogo político. Quizás lo que Laursen denomina «puntos ciegos ${ }^{70}$ de su tolerancia pueden ser reconducidos en parte a la interferencia política en su exégesis teológica. A diferencia de otros teólogos de su época, que mantenían posiciones parecidas ${ }^{71}$, como fueron William Chillingworth y John Tollston, sus posiciones triunfaron políticamente.

La interpretación «correcta» de la Escritura que desvelará una «religión racional» es un paso fundamental para evitar tanto una conducta absolutista por parte del magistrado, que se vería en otro caso obligado a prohibir «falsas formas de religión», como es el caso del catolicismo; como para evitar una conducta imperialista por parte de la Iglesia. Ambas cosas se consiguen despolitizando la religión interpretativamente, es decir, desinstitucionalizando a la Iglesia y reduciéndola a una mera asociación entre otras: algo que depende muy particularmente de la interpretación del pecado, del mundo de la gracia, de la ritualidad y el culto. Esto es lo que Locke sin duda consigue con su interpretación, y ello, sin desprenderse de una pieza fundamental en su sistema moral y político: la religión.

\footnotetext{
68 Abellán 2008: XXXVI.

69 Sobre la tolerancia cf. Marshall 2006; Herrero 2016; Tuckness 2002; Mitchell 1990: 64-83; Sherlock 1997: 19-49.

70 Cf. Laursen 2011: 25-42.

71 Cf. Prieto López 2017: XXXII.
} 


\section{BIBLIOGRAFÍA}

Abellán, J. 2008. «Estudio preliminar», en M. Lutero, Escritos políticos: IX-XXXVII. Madrid: Tecnos.

Ashcraft, R. 1969. "Faith and Knowledge in Locke's Philosophy», en J. W. Yolton (ed.), John Locke: Problems and Perspectives: 194-223. New York: Cambridge University Press.

Balaguer, V. 2017. "La Sagrada Escritura 'una cum Sacra Traditione' ante el reto de la 'sola Scriptura'». Scripta Theologica 49: 171-192.

Dunn, J. 1969. The political Thought of John Locke. An Historica Account of the Argument of the 'Two Treatises of Government'. London: Cambridge University Press.

Euben, R. 1999. Enemy in the Mirror: Islamic Fundamentalism and the Limits of Modern Rationalism. Princeton: Princeton University Press.

García Alonso, M. 2008. La teología política de Calvino. Barcelona: Anthropos.

García-Alonso, M. 2016. "Estudio preliminar», en Calvino, Escritos políticos: IX-LXIX. Madrid: Tecnos.

Gregory, B. S. 2012. The Unintended Reformation. How Religious Revolution Secularized Society. Cambridge, Masachusetts: The Belknap Press of Harvard University Press.

Hagen, K. 1993. Luther's Approach to Scripture as seen in his 'Commentaries' on Galatians 1519-1538. Tübingen: J. C. B. Mohr/ Paul Siebeck.

Hahn, S. W. y Wiker, B. 2013. Politicizing the Bible: The Roots of Historical Criticism and the Secularization of Scripture 1300-1700. New York: The Crossroad Publishing Company.

Herrero, M. 2015. La política revolucionaria de John Locke. Madrid: Tecnos.

Herrero, M. 2016. "The Quest for Locke's Political Theology». Ethics \& Politics XVIII: 83-109.

Herrero, M. 2017. "The Early Modern 'Philosophical Bible' and the Supposedly Secular Modern State». The European Legacy 22: 31-49.

Israel, J. 2001. Radical Enlightenment. Philosophy and the Making of Modernity 1650-1750. Oxford: Oxford University Press.

Kahn, P. 2011. Political Theology: Four New Chapters on the Concept of Sovereignty. New York: Columbia University Press.

Laursen, J. C. 2011. «Puntos ciegos en la teoría de la tolerancia: Milton, Spinoza, Locke y Bayle», en M.a J. Villaverde Rico y J. C. Laursen, Forjadores de tolerancia: 25-42. Madrid: Tecnos.

Lee, M. J. 2013. The Erosion of Biblical Certainty. Battles over Authority and Interpretation in America. New York: Palgrave/MacMillan.

Legaspi, M. 2010. The Death of Scripture and the Rise of Biblical Studies. Oxford: Oxford University Press.

Locke, J. 1824. "A Paraphrase and Notes on the Epistle of St Paul to the Galatians, Chorintians, Romans, Ephesians, to which is prefixed an Essay for the Understanding of St Paul Epistles by Consulting St Paul Himself», en J. Locke, The Works of John Locke in Nine Volumes: vol. 8. London: Rivington.

Locke, J. 1956. Ensayo sobre el entendimiento humano. México: Fondo de Cultura Económica.

Locke, J. 1967. Two Tracts on Government. Cambridge: Cambridge University Press

Locke, J. 1992. La conducta del entendimiento y otros ensayos póstumos. Anthropos: Barcelona.

Locke, J. 2002a. «Infallibility», en J. Locke, Writings on Religion: 69-73. Oxford: Clarendon Press.
Locke, J. 2002b. "A Vindication of the Reasonableness of Christianity», en J. Locke, Writings on Religion: 211-227. Oxford: Clarendon Press.

Locke, J. 2002c. «Ecclesia» en J. Locke, Writings on Religion: 80-81. Oxford: Clarendon Press.

Locke, J. 2007. La ley de la naturaleza. Madrid: Tecnos.

Locke, J. 2017. La razonabilidad del cristianismo. Madrid: Tecnos.

Lutero, M. 1883-1929. "Assertio omnium articulorum», en Werke. Weimarer Ausgabe 7: 98. Weimar: H. Böhlaus Nachfolger.

Marshall, J. 1994. John Locke: Resistance, Religion and Responsibility. Cambridge: Cambridge University Press.

Marshall, J. 2006. J. Locke, Toleration and Early Enlightenment Culture. Religious Intolerance and Arguments for Religious Toleration in Early Modern and Early Enlightenment Europe. New York: Cambridge University Press.

Mitchell, J. 1990. "John Locke and the Theological Foundation of Liberal Toleration: A Christian Dialectic of History». The Review of Politics 52: 64-83.

Mitchell, J. 1993. Not by Reason alone. Religion, History and Identity in Locke's Political Thought. Chicago: Chicago University Press.

Nuovo, V. 2002. «Introduction», en J. Locke. Writings on religion: XV-LVII. Oxford: Clarendon Press.

Nuovo, V. 2011. Christianity, Antiquity, and Enlightenment. Dordrecht: Springer Science Business Media B. V.

Nuovo, V. 2012. "General Introduction», en J. Locke, Vindications of the Reasonableness of Christianity: XIX-LXXVII. Oxford: Clarendon Press.

Pangle, T. 1988. The Spirit of Modern Republicanism: The Moral Vision of the American Founders and the Philosophy of Locke. Chicago: University of Chicago Press.

Prieto López, L. J. 2017. «Estudio introductorio», en J. Locke, La razonabilidad del cristianismo: XI-CXXXII. Madrid: Tecnos.

Pritchard, E. 2014. Religion in Public. Locke's Political Theology. Stanford: Stanford University Press.

Reventlow, H. G. 1985. The Authority of the Bible and the Rise of the Modern World. Philadelphia: Fortress Press.

Schwartzman, M. 2005. "The Relevance of Locke's Religious Arguments for Toleration». Political Theory 33: 678-705.

Sherlock, R. 1997. "The Theology of Toleration: A Reading of Locke's The Reasonableness of Christianity». Jewish Political Studies Review 9: 19-49.

Sommerville, J. C. 1992. The Secularization of Early Modern England. New York: Oxford University Press.

Spellman, W. M. 1988. John Locke and the Problem of Depravity. Oxford: Clarendon Press.

Tuckness, A. 2002. Locke and the Legislative Point of View. Toleration, Contested Principles, and the Law. Princeton: Princeton University Press.

Von Leyden, W. 1956. «John Locke and Natural Law». Philosophy 31: 26-35.

Waldron, J. 2002. God, Locke and Equality. Christian Foundations in Locke's Political Thought. New York: Cambridge University Press.

Wensheimer, J. C. 1993. Eighteenth Century Hermeneutics. Philosophy of Interpretation in England from Locke to Burke. New Haven \& London: Yale University Press.

Zuckert, M. 2002. Launching Liberalism: On Lockean Political Philosophy. Lawrence: University Press of Kansas. 